Chuanqiang Chen $\cdot \mathrm{Lu} \mathrm{Xu} \cdot$ Dekai Zhang

\title{
Erratum to: The interior gradient estimate of prescribed Hessian quotient curvature equations
}

Published online: 17 August 2021

Erratum to: Manuscripta Math. (2017)

https://doi.org/10.1007/s00229-016-0877-4

In this note we correct a typo in the proof of the main result of [1], that is (3.10).

(3.10) should be

$$
F^{1 i} u_{1 i}=\frac{\partial F}{\partial a_{1 i}} \frac{1}{W^{2}} u_{1 i}=\frac{\partial F}{\partial a_{1 i}} a_{i 1} \leq 0 .
$$

The last inequality in the above fomula holds from the concavity of $\left[\frac{\sigma_{k}}{\sigma_{l}}\right]^{\frac{1}{k-l}}$. We can give a detailed proof as follows. Following the proof in [1], we assume $A=\left\{a_{i j}\right\}_{n \times n}$ with $a_{i j}=a_{j i}$, and at $x_{0}$, we have $a_{i j}=0$ for $2 \leq i \neq j \leq n$. For any $i_{0} \geq 2$, consider

$$
g(t)=\left[\frac{\sigma_{k}(B+t C)}{\sigma_{l}(B+t C)}\right]^{\frac{1}{k-l}}, \quad(-1 \leq t \leq 1),
$$

where $B=\left\{b_{i j}\right\}_{n \times n}$ with $b_{1 i_{0}}=b_{i_{0}}=0$ and $b_{i j}=a_{i j}$ otherwise, and $C=$ $\left\{c_{i j}\right\}_{n \times n}$ with $c_{1 i_{0}}=c_{i_{0} 1}=a_{i_{0} 1}$ and $c_{i j}=0$ otherwise. Then we have $B+C=A$.

Since $\sigma_{1}(B+t C)=\sigma_{1}(B)$ and

$$
\sigma_{m}(B+t C)=\sigma_{m}(B)-t^{2} a_{1 i_{0}}^{2} \sigma_{m-2}\left(B \mid 1 i_{0}\right)
$$

for $m \geq 2$, we can get

$$
\begin{aligned}
\sigma_{m}(B+t C) & =\sigma_{m}(A)+\left(1-t^{2}\right) a_{1 i_{0}}^{2} \sigma_{m-2}\left(B \mid 1 i_{0}\right) \\
& =\sigma_{m}(A)+\left(1-t^{2}\right) a_{1 i_{0}}^{2} \sigma_{m-2}\left(A \mid 1 i_{0}\right)
\end{aligned}
$$

The original article can be found online at https://doi.org/10.1007/s00229-016-0877-4.

C. Chen: Department of Applied Mathematics, Zhejiang University of Technology, Hangzhou 310023, Zhejiang Province, China

e-mail: chuanqiangchen@zjut.edu.cn

L. Xu ( $\varangle$ ): School of Mathematics, Hunan University, Changsha 410082, Hunan Province, China

e-mail: xulu@hnu.edu.cn

D. Zhang: School of Mathematical Sciences, University of Science and Technology of China, Hefei, Anhui Province, China

e-mail: dekzhang@mail.ustc.edu.cn 


$$
\geq \sigma_{m}(A)
$$

for $t \in[-1,1]$ and $\sigma_{m}(B+t C)=\sigma_{m}(B-t C)$, which can also be obtained from

$$
\left|\begin{array}{cccc}
a_{11} & \cdots & t a_{1 i_{0}} & \cdots \\
\vdots & \ddots & & \\
t a_{i_{0} 1} & & a_{i_{0} i_{0}} & \\
\vdots & & & \ddots
\end{array}\right|_{m \times m}=\left|\begin{array}{cccc}
a_{11} & \cdots & -t a_{1 i_{0}} & \cdots \\
\vdots & \ddots & & \\
-t a_{i_{0} 1} & & a_{i_{0} i_{0}} & \\
\vdots & & & \ddots
\end{array}\right|_{m \times m} .
$$

Hence we know the eigenvalues of $B+t C$ are in the convex cone $\Gamma_{k}$ for $t \in[-1,1]$ and $g(-1)=g(1)$. From the concavity of $\left[\frac{\sigma_{k}}{\sigma_{l}}\right]^{\frac{1}{k-l}}$ in $\Gamma_{k}$, we have $g(t)$ is concave with respect to $t \in[-1,1]$. Hence

$$
0 \geq g^{\prime}(1)=\frac{1}{k-l}\left[\frac{\sigma_{k}(A)}{\sigma_{l}(A)}\right]^{\frac{1}{k-l}-1}\left[\frac{\partial \frac{\sigma_{k}(A)}{\sigma_{l}(A)}}{\partial a_{1 i_{0}}} a_{i_{0}}+\frac{\partial \frac{\sigma_{k}(A)}{\sigma_{l}(A)}}{\partial a_{i_{0}} 1} a_{1 i_{0}}\right] .
$$

Hence $\frac{\partial F}{\partial a_{1 i_{0}}} a_{i_{0}} \leq 0$ for any $i_{0} \geq 2$.

\section{References}

[1] Chen, C., Xu, L., Zhang, D.: The interior gradient estimate of prescribed Hessian quotient curvature equations. Manuscripta Math. 153(1-2), 159-171 (2017). https://doi.org/10. 1007/s00229-016-0877-4

Publisher's Note Springer Nature remains neutral with regard to jurisdictional claims in published maps and institutional affiliations. 